\title{
Electrical Conductivity for Irradiated, Grafted Polyethylene and Grafted Polyethylene with Metal Complex
}

\author{
A.H. Ashour, H.M. Saad, M.M. Ibrahim \\ Radiation Physics Dept., National Center for Radiation \\ Research \& Technology - Nasr City, Cairo, Egypt
}

The effect of silver metal (Ag) in DC conductivity of high density polyethylene (HDPE) and low density polyethylene (LDPE) grafted films with acrylic acid (AAC) at different percentage 95, 120 and 160\% has been investigated. It is also aimed to perform an extensive study on the electrical properties of (LDPE) and (HDPE) before and after irradiation by $\gamma$-rays at different environment in order to understand the nature of the conduction mechanism and charge transport in these materials. The results show a substantial increase in the electrical conductivity $(\sigma)$ for both types of polyethylene exposed to $\gamma$-irradiation in air and in vacuum at dose range $(0-500 \mathrm{kGy})$. The improvement in $\sigma$ for LDPE in oxygen is more pronounced than HDPE. Thermal activated conduction mechanism is predominant for both $L D P E$ and HDPE at different $\gamma$-doses in air, and in oxygen. The grafting with PAAc causes an increase in the conductivity by approximately six order higher than that of ungrafted films. Grafted copolymer was obtained by adding metal salt solution $\mathrm{AgNO}_{3}$ to both graphted LDPE and HDPE at $373 \mathrm{~K}$ for $15 \mathrm{hrs}$. Increasing of Ag ions in the copolymer samples at room temperature leads to a slight decrease in the $\sigma$ value.

\section{Introduction:}

Recently, polymer-metal complexes have become of great interest because of their unique properties useful in some industrial applications [1-4]. For this purpose it has been possible to modify polymers by grafting monomers onto the surface, or throughout the bulk of the original in order to produce copolymer which has properties more suitable for a given application. Polyethylene grafted with acrylic acid using $\gamma$-ray initiation, has a result which is semi-permeable and useful for a variety of functions, e.g. as a separator in alkaline battery systems and field of semiconductors. 
The present work, involves studying of electrical conductivity for the grafted copolymer with metal complex and irradiated polymer at different environment as a function of temperature. The main objective of this work was to examine systematically the influence of the silver nitrate $\mathrm{AgNO}_{3}$ content on the electrical properties of both LDPE and HDPE. The observed results are discussed relative to the role of environment medium, $\gamma$-irradiation and $\mathrm{Ag}$ ion in the grafted polymer.

\section{Experimental Procedure:}

\subsection{Materials}

Commercial low density polyethylene (LDPE) films of thickness 0.66 $\mathrm{mm}$ and high density polyethylene (HDPE) films of thickness $1.46 \mathrm{~mm}$ were provided by El-Sherief for plastics industrial, Cairo, Egypt. The films were washed with acetone and dried in vacuum oven at $50^{\circ} \mathrm{C}$. Acrylic acid (AAc) (Aldrich) of purity 99\% was used without further purification. Inhibior: Ferric chloride $\left(\mathrm{FeCl}_{3}\right)$, Solvent: bidstilled water, Metal Salt: Silver nitrate $\left(\mathrm{AgNO}_{3}\right)$ were used as received.

\subsection{Grafting process}

Strips of LDPE and HDPE films were weighed and then immersed in aqueous AAc glass ampoules and Ferric chloride was added to the reaction medium to minimize homopolymerization during the radiation grafting process. The direct $\gamma$-irradiation grafting was carried out at dose $20 \mathrm{kGy}$ (dose rate $3 \mathrm{kGy} / \mathrm{h}$ ) under nitrogen atmosphere. The grafted-films were removed and washed thoroughly with distilled water. For instance, poly (acrylic acid) (PAAc) was removed by washing grafted films thoroughly with hot distilled water and soaked over night in water to extract the residual monomer and the homopolymer which may be occured in the film and then weighed. The grafting percentage was determined by the percent increase in weight; grafting $\%=100$ $\left[\left(\mathrm{W}_{\mathrm{g}}-\mathrm{W}_{\mathrm{o}}\right) / \mathrm{W}_{\mathrm{o}}\right]$, where $\mathrm{W}_{\mathrm{o}}$ and $\mathrm{W}_{\mathrm{g}}$ represent the weights of initial and grafted films, respectively.

\subsection{Graft copolymer doped metal}

The different grafting copolymer doped metal were prepared by refluxing the grafted LDPE and HDPE films with 1 and 10\% wt.\% of silver nitrate $\left(\mathrm{AgNO}_{3}\right)$ solution for $20 \mathrm{hr}$ at $100^{\circ} \mathrm{C}$. The treated grafted films were washed several times using hot distilled water and then dried under vacuum in oven at $50^{\circ} \mathrm{C}$.

The X-Ray Fluorescence (XRF) technique, (hnu, PC TEFA, Tube Fluorescence Analyzer System, USA) was used to confirm the existence of doped metal in the graft copolymer. 


\subsection{Electrical measurement}

DC Conductivity of PE film was measured by a two probe technique. Good electrical contact on both faces of the PE film was prepared by silver electrodes. A coaxial shielded cable was used to eliminate electric noise in the case of low current measurements. The used electrometer was Keithly programmable electrometer model 617. The temperature of the film was recorded with chromel-aluminel thermocouple kept in close thermal contact with film surface.

\section{Results and Discussion:}

\subsection{Temperature Dependence of Unirradiated Samples}

Much attention has been given lately to the study of electrical properties of charge transfer complexes in polymer [5]. Figure (1) depicts the temperature dependence of the electrical conductivity $\sigma$ of the unirradiated low density polyethylene LDPE and high density polyethylene HDPE samples respectively. It is clear from the figure that $\sigma$ for LDPE has an independent behaviour on temperature in the range 303-348K then sharply increases with temperature (>348K). Meanwhile, a two stages of behviour with two different slopes are distinguished in the case of HDPE sample. The activation energies in both stages (for both samples) are tabulated in the table $(1 \& 2)$ respectively using the Arrhenius equation:

$$
\sigma=\sigma_{0} \exp -\Delta \mathrm{E} / \mathrm{K} \mathrm{T}
$$

which governs the $\log \sigma$ against 1000/T (K) relations, where $\sigma$ and $\sigma_{0}$ are the conductivity and initial conductivity respectively, $\mathrm{K}$ is the Boltzmann constant and $\Delta \mathrm{E}$ is the activation energy.
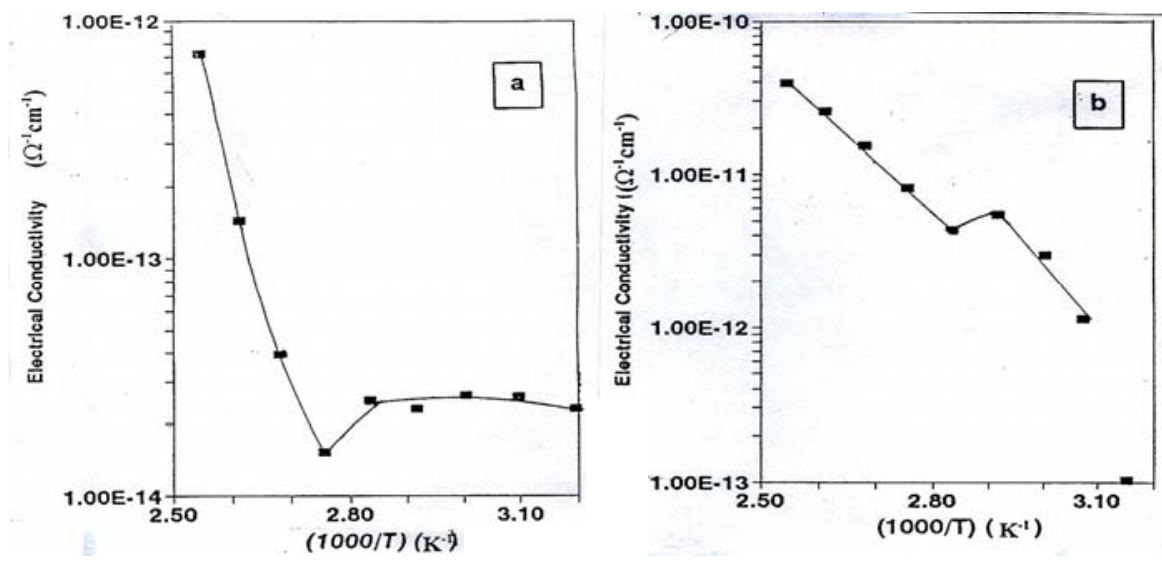

Fig. (1) : Electrical conductivity of unirradiated PE: a) LDPE and b) HDPE. 


\subsection{Dose dependence of the electrical conductivity}

The objective of the irradiation of polymer is usually the modification of their properties such as mechanical, electrical and physicochemical. In fact, the chemical changes involved in these irradiated polymers are relatively small. However, such minor chemical changes may produce such significant physical modification [6]. Thus radiation induced modification of polymers provides an extremely valuable method for the study in polymer science.

Figures (2 and 3) show the relation between the electrical conductivity $\sigma$ and the $\gamma$-irradiation dose irradiation measured at $30^{\circ} \mathrm{C}$ of LDPE and HDPE at different environmental medium.

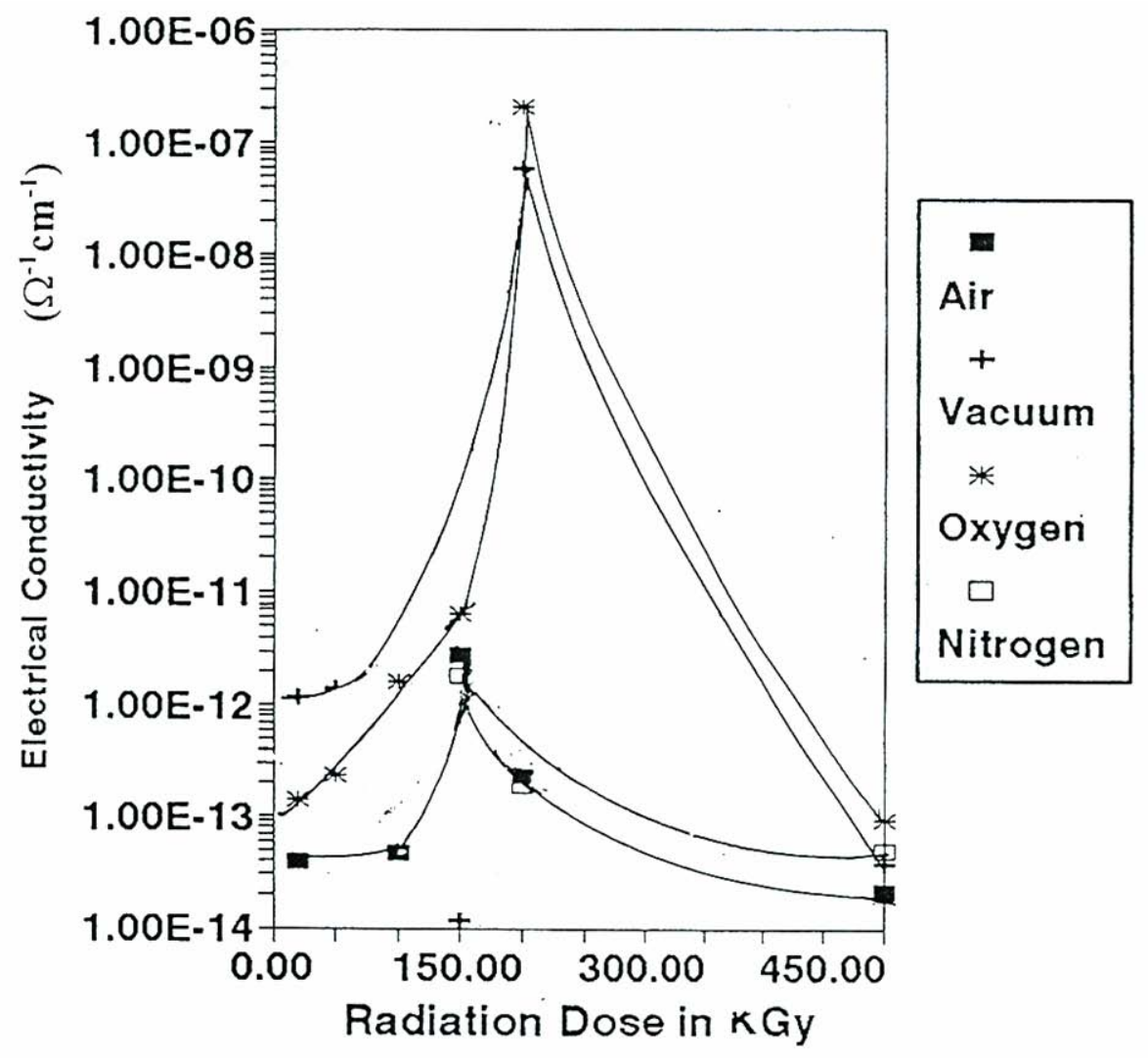

Fig. (2): Electrical conductivity at room temperature of irradiated LDPE at different atmospheres. 




Fig. (3): Electrical conductivity at room temperature of irradiated HDPE at different atmospheres and irradiation dose.

It is clear that the variation of $\sigma$ with irradiation dose markedly depends on the type of PE and the environmental medium. It may be presumed that the action of gamma rays results in excitations of its molecules and creation of free electrons and ions that migrate through the polymer network till they are trapped somewhere, leaving deficient regions. These electronic and ionic configurations cause the changes in the electric conductivity of both LDPE and HDPE samples. Generally, the major effects in polymers arise from the dissociation of primary valence bonds into radicals [7].

The dissociation of $\mathrm{C}-\mathrm{C}$ and $\mathrm{C}-\mathrm{H}$ bonds leads to different results; degradation and crosslinking that may occur simultaneously as clearly detected for LDPE in different environmental medium except for LDPE surrounded by nitrogen. Crosslinking is the predominate reaction on the irradiation of HDPE in air and vacuum. Degradation in the host material of LDPE samples was obviously detected for LDPE sample in nitrogen. In addition, from Figs. (2, 3) one could observe that the electrical conductivity $\sigma$ is improved with irradiation in different environmental atmospheres \{especially in vacuum and air for HDPE and in oxygen for LDPE $(\leq 115 \leq 225 \mathrm{kGy})\}$. This behaviour confirms the conclusion reported by Plans [8], which concluded that the film of polyethylene shows a substantial increase in electrical conductivity when it is exposed to a bromine atmosphere at room temperature. Thus in order to participate in charge transfer, electrons or holes must jump from a chain to a neighbouring one. However, transfer between chains is admittedly slow, leading 
to very low mobilities ( $\cong 10^{-12} \mathrm{~cm}^{2} / \mathrm{VS}$ ). A way to improve the mobility level is to dope PE with electron- donating molecules. In this case, reaction occurs predominantly at the fold surface [9].

The improvement in $\sigma$ for LDPE in oxygen is more pronounced than HDPE because of the fact that the conductivity $\sigma$ value is undoubtedly connected with the maximum possible amount of $\mathrm{O}_{2}$ which can accommodated in the non-crystalline regions. Hence one could systematically vary the conductivity of these PE samples over a large range, by doping samples with varying non-crystalline contents [10].

\subsection{Temperature dependence of irradiated samples.}

Polymers are generally divided into those that predominantly crosslink and those which predominantly degrade. Polyethylene belongs to the first category [5].

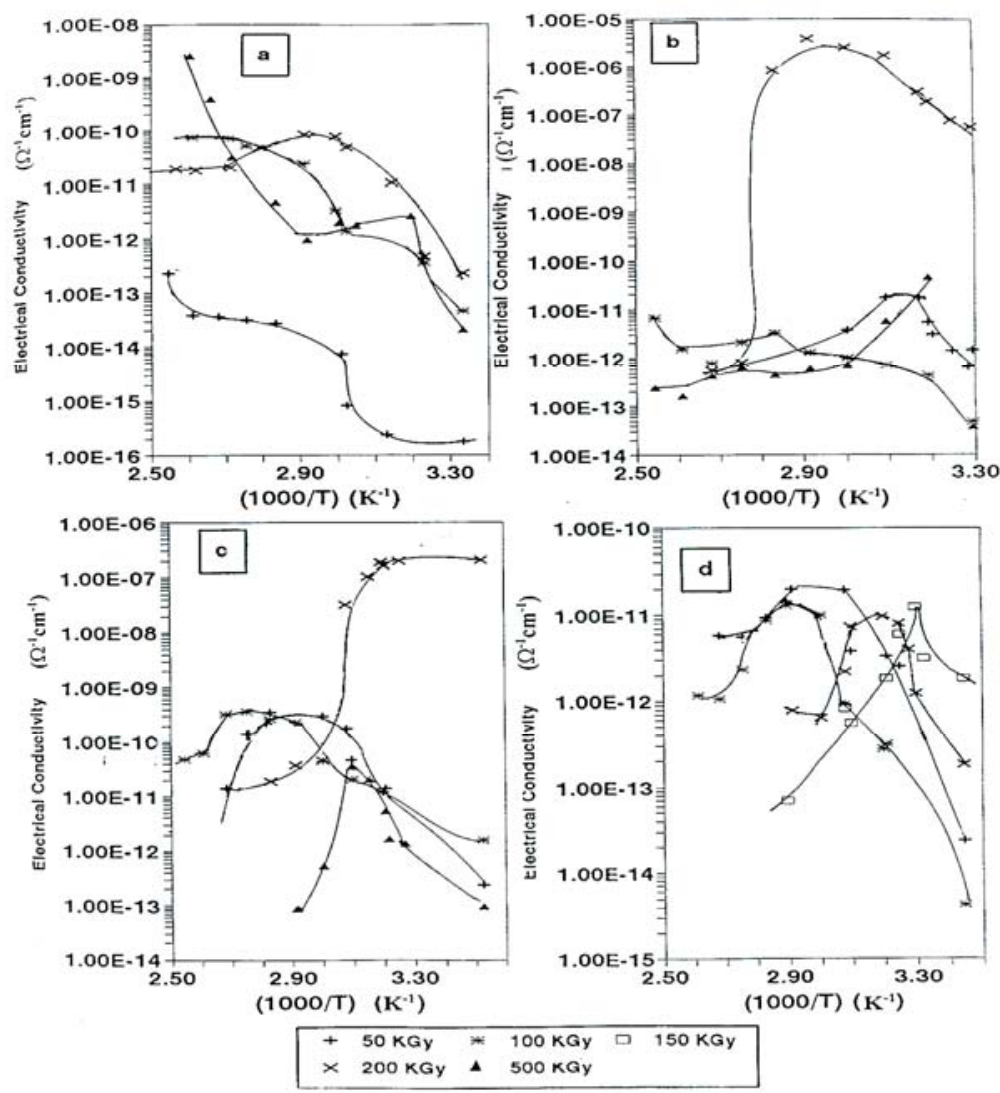

Fig. (4): Electrical conductivity of irradiated LDPE at different atmospheres; a : air, b : vacuum, c : oxygen \& d : nitrogen 


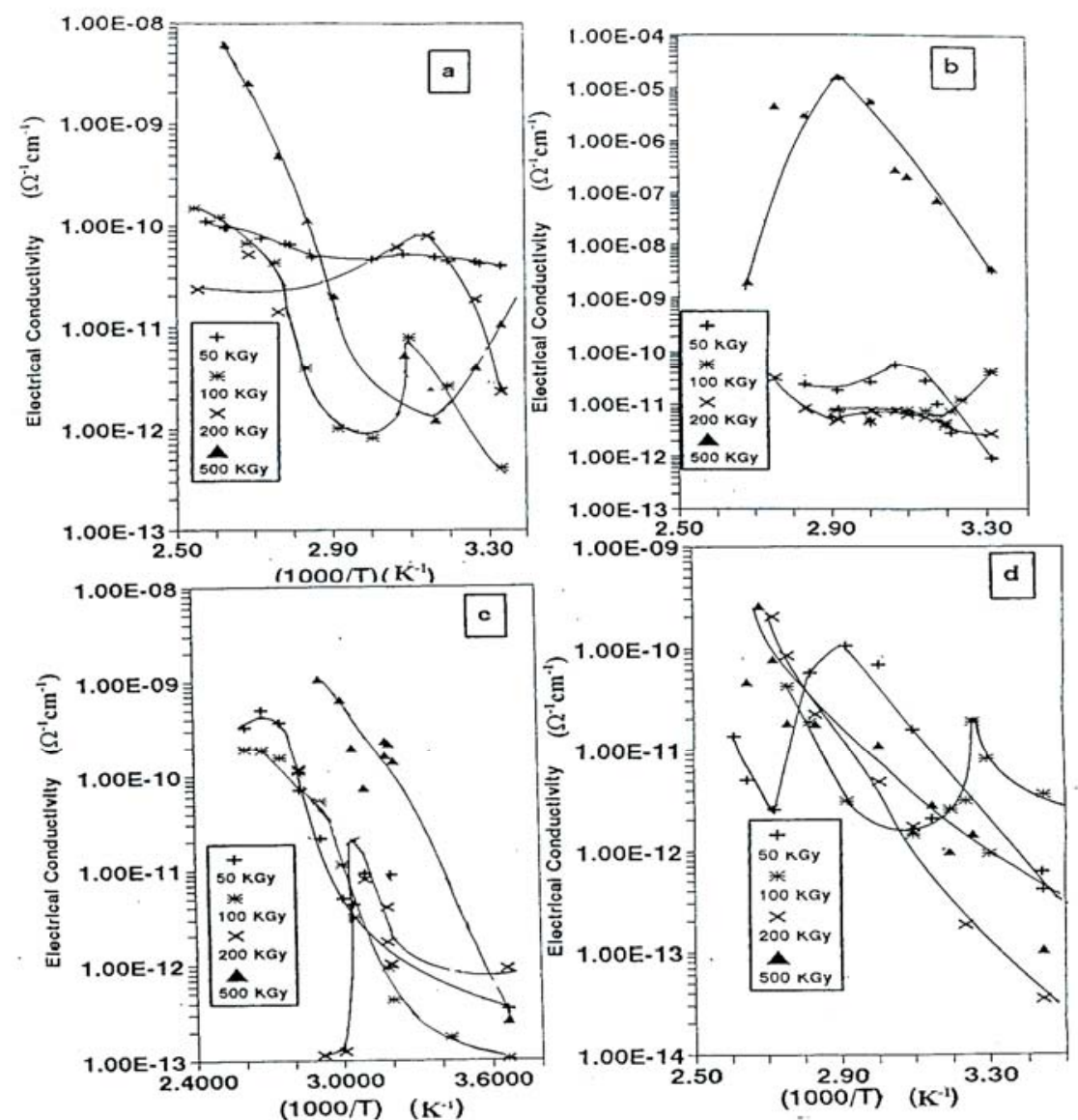

Fig. (5) : Electrical conductivity of irradiated HDPE at different atmospheres:

a) air, b) vacuum, c) oxygen and d) nitrogen.

Figures (4 and 5) depict the temperature dependence of the electrical conductivity $\sigma$ for LDPE and HDPE polymer samples at different environmental atmospheres and $\gamma$-doses. The function $\log \sigma=\mathrm{f}(1 / \mathrm{T})$ can be represented by one or two least square fitting lines depending on atmosphere and the dose, indicating the type of conduction according to Mott [11]. The obtained data indicate that the activation energy is highly affected by both the environmental medium and $\gamma$-dose as detected from.

\subsection{Electrical conductivity of grafted PE}

In recent years, polymer-metal complexes have become of great interest because their electrical, thermal and mechanical properties, are useful in some industrial applications and biological materials [1, 12- 13]. For acrylic acid 
monomer which polymerizes quickly under radiation initiation, its crosslinked polymers are known to form stable complexes with transition metal ions but they often absorb the metal ions slowly because of relatively slow diffusion of ions into the polymer [14].

Graft copolymer was prepared by the direct radiation graft copolymerization of aqueous acrylic acid onto thick LDPE and HDPE films. Grafted LDPE and HDPE films, having different degrees of grafting (99.39, 147.75 and $162.65 \%$ ) and (95.12, 121.35 and 160.95\%), respectively, were obtained and used for this study. Meanwhile, the different copolymer Ag complexes were prepared by refluxing the LDPE and HDPE grafted (with different degrees) films with 1.0 and 10.0 wt $\%$ metal salt solution $\mathrm{AgNO}_{3}$ for $15 \mathrm{hr}$ at $100^{\circ} \mathrm{C}$.

Figure (6) shows the electrical conductivity of the original LDPE and HDPE as well as the grafted film as a function of temperature. It can be seen that, the grafted copolymers possess electrical conductivity $(\sigma)$ of approximately one million $\left(10^{6}\right)$ times higher than that of LDPE or HDPE original material at $303 \mathrm{~K}$.
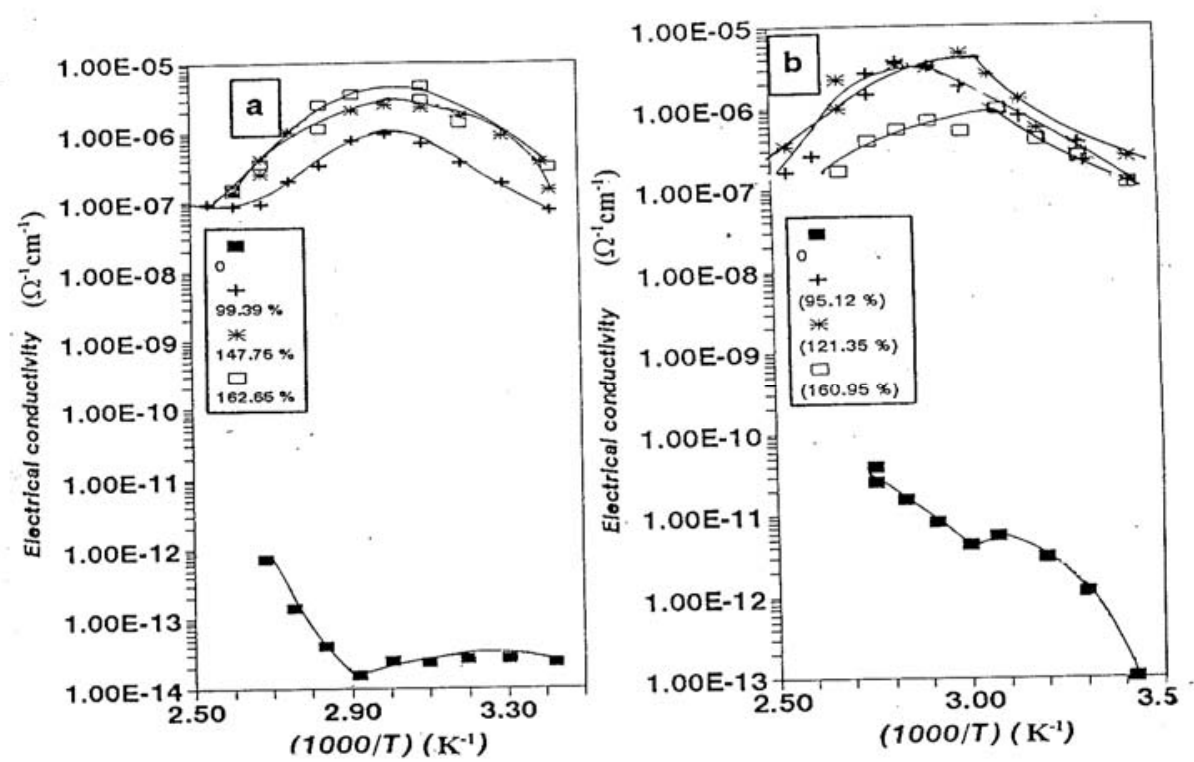

Fig. (6) : Electrical conductivity of PE-g-PAAc with different percentage of grafting: a) LDPE and b) HDPE.

The value of $\sigma$ increases slightly with the degree of grafting for both LDPE and HDPE. The increase of $\sigma$ for LDPE and HDPE grafted with AAc may be due to the free mobility of carboxylic acid groups $(\mathrm{COOH})$ of the graft 
chains. The effect of ionizing radiation on the polymer substrate during grafting process must also be taken into consideration and its influence on electrical properties is included $[15,16]$.

Figures (7 and 8) show the electrical conductivity of both LDPE and HDPE-grafted films doped with $\mathrm{AgNO}_{3}$ metal salt solution (1 and $10 \mathrm{wt} \%$ ) as a function of elevated temperature.
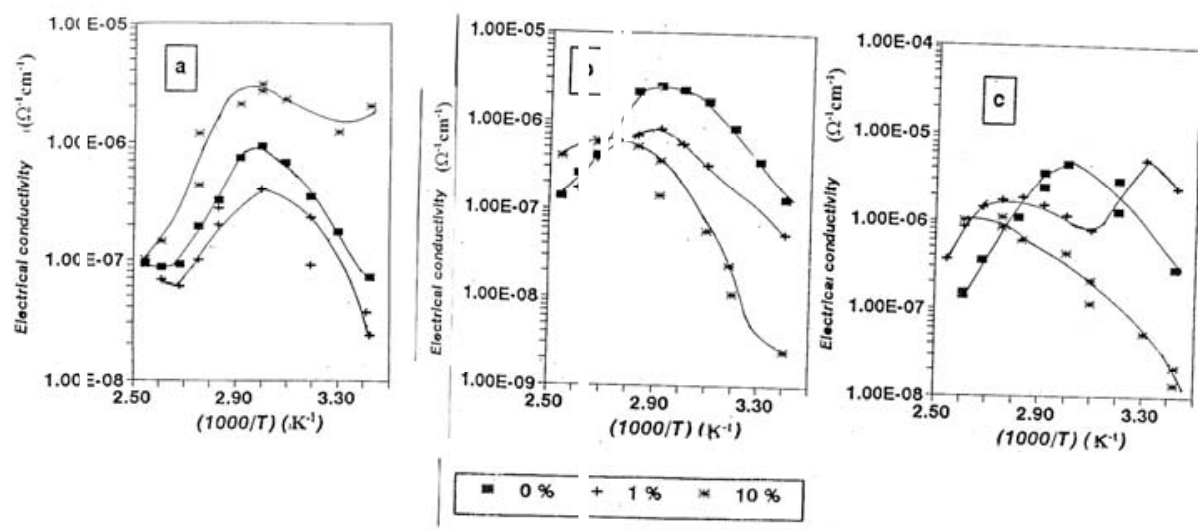

Fig. (7) : Electrical conductivity of LDPE-a-PAAc complexed with different percentage Of $\mathrm{Ag}+$ ion having the various grafting yields:

a) $99.39 \%$, b) 147.75 and c) $162.65 \%$.


Fig. (8) : Electrical conductivity of grafted HDPE with different percentage of $\mathrm{AgNO}_{3}$ (additive), metal complexes and grafting ratios: a) 95.12\%, b) 121.35 and c) $160.95 \%$.

It can also be seen that, the grafted LDPE and HDPE copolymers that doped with $\mathrm{Ag}$ ions possess electrical conductivity $(\sigma)$ of one million $\left(10^{6}\right)$ times greater than that of both HDPE and LDPE original materials at $303 \mathrm{~K}$ (ef. Fig. $1 \mathrm{a}$ and b). Increasing the weight percentage of Ag ions leads to a slight decrease in the $\sigma$ value at $303 \mathrm{~K}$ for grafted LDPE (at 147.35 and $162.65 \%$ 
degree of grafting) and for HDPE at (95.12 \& $121.35 \%$ degree of grafting). So, doping by Ag ions does not have a major effect on the electrical conductivity values of both grafted LDPE and HDPE polymers. The decreasing behaviour in $\sigma$, for both grafted LDPE and HDPE doped and undoped with metal ions, above certain temperature (ranging from 320-340 K depending on both degree of grafting and metal ion weight percent) can be reasonably explained by the formation of new phase transition in the copolymer structure above such temperature.

The calculated values of activation energy are given in Tables (1 and 2). The values of the activation energy for grafted undoped and metal doped copolymers are lower than that of the original LDPE and HDPE polymer ungrafted one.

Table (1) : Activation energy for LDPE grafted copolymerand graft copolymer containing Ag metal

\begin{tabular}{|l||c|c|c||}
\hline \multicolumn{1}{|c||}{ Sample } & $\mathbf{G}(\mathbf{\%})$ & AgNO $_{\mathbf{3}} \mathbf{( \% )}$ & $\Delta \mathbf{E}(\mathbf{e V})$ \\
\hline \hline LDPE & Blank & - & 1.80 \\
LDPE-g-PAAc & 99.39 & - & 0.57 \\
LDPE-g-PAAc & 145.75 & - & 0.75 \\
LDPE-g-PAAc & 162.65 & - & 0.56 \\
LDPE-g-PAAc/Ag & 99.39 & 1 & 0.76 \\
LDPE-g-PAAc-Ag & 99.39 & 10 & 0.61 \\
LDPE-g-PAAc-Ag & 147.75 & 1 & 0.57 \\
LDPE-g-PAAc-Ag & 147.75 & 10 & 0.86 \\
LDPE-g-PAAc-Ag & 162.65 & 1 & 0.30 \\
LDPE-g-PAAc-Ag & 162.65 & 10 & 0.67 \\
\hline
\end{tabular}


Table (2): Activation energy for HDPE grafted one with acrylic acid (AAc) and graft copolymer containing Ag metal ion

\begin{tabular}{||l|c|c|c||}
\hline \multicolumn{1}{|c|}{ Sample } & $\mathbf{G}(\mathbf{\%})$ & AgNO $_{\mathbf{3}}(\mathbf{\%})$ & $\Delta \mathbf{E}(\mathbf{e V})$ \\
\hline \hline HDPE & Blank & - & 0.62 \\
HDPE-g-PAAc & 95.12 & - & 0.62 \\
HDPE- g-PAAc & 121.35 & - & 0.64 \\
HDPE- g-PAAc & 160.95 & - & 0.55 \\
HDPE-g-PAAc-Ag & 95.12 & 1 & 1.23 \\
HDPE-g-PAAc-Ag & 95.12 & 10 & 0.61 \\
HDPE- g-PAAc-Ag & 121.35 & 1 & 0.70 \\
HDPE- g-PAAc-Ag & 121.35 & 10 & 0.61 \\
HDPE- g-PAAc-Ag & 160.95 & 1 & 0.82 \\
HDPE- g-PAAc-Ag & 160.95 & 10 & 0.79 \\
\hline
\end{tabular}

\section{Conclusion:}

The effect of modification of LDPE and HDPE with grafting of acrylic acid and doped silver metal ion on the electrical properties has been described. Moreover, the radiation effects on the electrical properties under different environments of pure and modified LDPE and HDPE have also been depicted. It can be concluded from the above studies that: LDPE and HDPE samples show a substantial increase in electrical conductivity $(\sigma)$ when they were exposed to $\gamma$-irradiation in air and in vacuum in the dose range (0-150 kGy). The improvement in $\sigma$ for LDPE in oxygen is more pronounced than HDPE. The grafting of LDPE and HDPE films with AAc possesses the electrical conductivity $(\sigma)$ of approximately one million $\left(10^{6}\right)$ times higher than that of ungrafted LDPE and HDPE samples. Increasing the weight percentage of Ag ions in the grafted LDPE and HDPE samples leads to a slight decrease in the $\sigma$ value at $303 \mathrm{~K}$.

\section{References}

1. M. A. Abd-El-Ghaffar, E. A. Hegazy, A. M. Dessouki, and N. M. El-Sawy, $2^{\text {nd }}$ Arab International Conference on Polymer Materials, Alexandria, Egypt, (1990).

2. M. A. Abd-El-Ghaffar, E.A. Hegazy, A.M. Dessouki, N.B. El-Assy and N.M. El-Sawy, J. Radiat. Phys. Chem. 38, (4), 669 (1991). 
3. F. Abd-Rehim, A. A. Abdel-Fattah and F. A. Soliman, Arab. J. Sci. Eng., 19, 2A, 143 (1994).

4. I. M. Abramova, L. G. Kazaryan, V. A. Vatagina, V. A. Vasil'ev, N. I. Kuzina, V. S. Tikhomirov and V. I. Serenkov, Plast. Massy, 9, 45 (1980).

5. A. K. Woods and Pikaev, Applied Radiation Chemistry: Radiation Processing, John Wiley \& Sons; 110 (1994).

6. E. Witt, J. Polymer Sci., 41, 507 (1959).

7. F. W. Billmeyer, Jr.: "Text Book of Polymer Science", Wiley Intersci., Pub. New York, 373 (1971).

8. P. Plans and Balta F. J. Calleja, J. Polymer Bulletin 5, 311 (1981).

9. J. Guzman, J. G. Fatou and J. M. Perna, J. Macromolec. Chem., 181, 1051 (1980).

10. J. Martinez Salazar and F. J. Balta-Calleza, J. Crystal Growth, 48, 283 (1980).

11. N. F. Mott, and E. A. Davis, "Electronic Prosses in Non-Crystalline Materials”, Clarendon, Oxford (1979).

12. L. J. Paliwal and R. B. Kharat, J. Macromolec. Sci. Chem. A-26, 843 (1989).

13. P. W. Atkins "Physical Chemistry", Oxford University Press, New York (1978).

14. D. Braun, and H. Bouderka, Eur. Polym. J. 11, 561 (1975).

15. A. H. Ashour, M. B. S. Osman and S. M. Mokhtar, $3^{\text {rd }}$ Arab International Conference on Polymer Sci. Technology, Sep. 4 - 7, Mansoura University, Egypt, 29 (1995).

16. A. Charlesby, Atomic Radiation and Polymers, Pergamon, Press New York (1960). 